\title{
Zukunftswerkstatt der Ulrich Saxer-Stiftung zum Thema "Media Labs": Schickes Label oder modernes Arbeiten in der Kommunikationswissenschaft?
}

\author{
Corinne Schweizer, University of Zurich, IKMZ - Department of Communication and Media \\ Research, Switzerland \\ c.schweizer@ikmz.uzh.ch
}

Media Labs: Ist das nur ein schickes Label oder steht dahinter eine moderne Form der Arbeitsorganisation für die kommunikationswissenschaftliche Forschung? Dieser Frage ging die Zukunftswerkstatt der Ulrich Saxer-Stiftung nach, die am 23. Oktober 2020 an der Universität Zürich stattfand. Das Ziel der am IKMZ angesiedelten Stiftung ist es, den kommunikationswissenschaftlichen Nachwuchs in der Schweiz zu fördern. ${ }^{1}$ Das tut sie unter anderem durch eine jährliche Werkstatt zu einem aktuellen Thema. Unter der Leitung von Dr. Corinne Schweizer wurde die Werkstatt Coronabedingt als hybride Variante durchgeführt: Einige Teilnehmende und Referierende sassen im Hörsaal, andere waren online zugeschaltet.

Die Werkstatt startete mit dem Vortrag von Prof. Dr. Gudela Grote, Professorin für Arbeits- und Organisationspsychologie an der ETH Zürich. Aus arbeitspsychologischer Sicht ist es nicht entscheidend, ob sich ein Team «Lab» nennt oder nicht, denn das Arbeiten in der Gruppe ist unabhängig vom Label generell äusserst anforderungsreich. Gerade dann, wenn die Gruppe kreativ sein soll und unterschiedliche kulturelle Hintergründe aufeinandertreffen. Oft helfe der Faktor Zeit, um als Team zusammenzuwachsen, jedoch müssten Mitarbeitende heute in vielen Settings - z. B. im Medizinbereich oder im Flugverkehr - in ständig wechselnden Teams arbeiten. Um als Lab erfolgreich zu sein, riet Grote unter anderem zu einer klaren Rollenverteilung und einem partizipativen Führungsstil.

1 FürnähereInformationensiehehttps://www. ikmz.uzh.ch/de/department/networks/ saxer-foundation.html.
In einem zweiten Beitrag diskutierte Hans Danuser, renommierter Schweizer Fotograf, das Thema aus künstlerischer Perspektive. Danuser hat sich in seinem Projekt «In Vivo» während zehn Jahren mit Laboren beschäftigt und interpretierte diese als Orte, die mit grosser Macht und gesellschaftlichen Werten verknüpft sind. Entsprechend fotografierte er in Krankenhäusern, Kernkraftwerken und militärischen Einrichtungen und fokussierte dabei auf tabuisierte Dinge, die kaum bildlich dargestellt wurden. Danuser befasste sich aber auch mit der Architektur von Labs, und der Frage, wie die Zusammenarbeit räumlich gestaltet werden kann. Prof. Dr. Otfried Jarren, Präsident der Ulrich Saxer-Stiftung, führte das Gespräch und stellte einige Querbezüge zu Journalismus und Medienforschung her.

Online zugeschaltet war Prof. Dr. Gesa Ziemer, Professorin an der HafenCity Universität in Hamburg. Ziemer leitet das von der Stadt Hamburg finanzierte Science City Lab (SCL), das in Kooperation mit dem Media Lab des MIT Städte im Kontext der Digitalisierung erforscht. Das SCL ist inter- und transdisziplinär: Forschende aus zahlreichen Disziplinen (darunter jedoch bisher noch niemand aus der Kommunikationswissenschaft) versuchen in Zusammenarbeit mit Politik, Industrie und Mitgliedern der Zivilgesellschaft die Probleme der Hansestadt zu lösen und die öffentliche Diskussion zu versachlichen. Wie so etwas aussehen kann, zeigte Ziemer am «Finding Places» Projekt, wo Bürgerinnen und Bürger diskutierten, in welchen Gebieten der Stadt Flüchtlingsunterkünfte gebaut werden könnten. 
Im abschliessenden hybriden Panel trafen Vertretende von drei sehr unterschiedlichen «Media Labs» aufeinander. Das Media Technology Center der ETH Zürich ist ein Informatik-Lab, das von Schweizer Medienhäusern finanziert wird. Dr. Severin Klingler und sein Team entwickeln z.B. Spracherkennungssoftware oder «News Recommender Systems». Mit aktuellen Problemen in der Medienregulierungs-Praxis beschäftigt sich hingegen das von Dr. Gergana Baeva geleitete Media Policy Lab der Medienanstalt Berlin-Brandenburg (MABB). Das kleine "Behördenlab» sieht sich als Schnittstelle zwischen dem MABB und seiner Umwelt und organisiert den Austausch mit Wissenschaft und Praxis. Das Digital Democracy Lab der Universität Zürich ist schliesslich ein politikwissenschaftliches Lab, das sich mit der digitalen Transformation der Politik beschäftigt. Dafür haben Prof. Dr. Fabrizio Gilardi und seine Mitarbeitenden in den letzten 18 Monaten eine Forschungs- infrastruktur aufgebaut, die eine effiziente, skalierbare Datenerhebung und -analyse erlaubt und offen sein soll für andere Forschende.

Wer an der diesjährigen Zukunftswerkstatt dabei war, hat einen guten Eindruck davon bekommen, welche vielfältigen Erwartungen mit dem Begriff «Media Labs» verbunden sind. Ob hinter dem Label neue Arbeitsformen stecken, und welche das sind, muss aber im Einzelfall diskutiert werden. Die Zukunftswerkstatt hat zudem gezeigt, dass hybride Veranstaltungsformen funktionieren können. 2021 wird die Werkstatt - unter hoffentlich einfacheren Bedingungen - von Dr. Dominique Wirz (DCM, Universität Fribourg) organisiert. Sie übernimmt das Amt von der Autorin dieses Textes, die in den letzten Jahren fünf Workshops zu Themen wie Berichterstattungsfreiheit, Repräsentation von Frauen* und Migrant*innen oder Vertrauen in Medien und Politik konzipiert und durchgeführt hat. 